04

\title{
Волноводные моды в планарной мелкослоистой структуре графен-диэлектрик
}

\author{
(С) Д.А. Евсеев, Д.И. Семенцов \\ Ульяновский государственный университет, \\ 432970 Ульяновск, Россия \\ ฯ e-mail: sementsovdi@mail.ru \\ Поступило в редакцию 23.05.2017 г.
}

Исследованы особенности волноводного распространения мод в планарной структуре, состоящей из чередующихся слоев диэлектрика и графена. В рамках приближения эффективной среды получены дисперсионные соотношения для симметричных и антисимметричных волноводных мод. На основе их численного анализа построены частотные зависимости константы распространения и затухания, групповой и фазовой скоростей, энергетического потока, переносимого волноводными модами, проанализировано влияние доли графена в структуре на поведение волноводных мод.

DOI: $10.21883 /$ OS.2018.02.45530.129-17

\section{Введение}

В последние годы активно исследуются особенности взаимодействия с электромагнитным полем монослоев графена и слоистых структур на его основе. Уникальные электронные свойства графена - высокая подвижность носителей заряда и, как следствие, высокая проводимость при практическом отсутствии в широкой частотной области поглощения, возможность локализации волнового поля в очень тонких слоях и управляемость как электрическим полем, так и магнитным определяют особые его оптические свойства и делают одним из наиболее перспективных материалов фотоники и оптоэлектроники [1-7]. Так, фотонные кристаллы (ФК) с различным типом чередования слоев графена и диэлектрика перспективны для создания различных электрически управляемых устройств. Ввиду особенностей дисперсии проводимости графена и соответственно диэлектрической проницаемости (ДП) физические свойства ФК на основе графена могут существенно отличаться от свойств ФК на основе других материалов [8-11].

Для практических применений важную роль также играют направляющие свойства графеновых структур. В работе [12-14] была показана возможность распространения локализованных на монослое графена ТМи ТЕ-волн, дисперсия которых лежит в терагерцовой частотной области. Активно исследуется способность структуры, образованной двумя слоями графена с разделяющим тонким слоем диэлектрика, удерживать локализованные плазмонные моды, разрабатывается возможность создания устройств волноводного типа на основе структур из двух слоев графена [15-17].

В настоящей работе исследуются особенности распространения волноводных мод в планарной структуре, ограниченной двумя слоями графена, между которыми находится мелкослоистая среда из чередующихся слоев диэлектрика и графена. Для волноводных мод ТЕ-типа получены и проанализированы дисперсионные соотно- шения, построены распределения волнового поля в структуре, найдены групповые и фазовые скорости мод.

\section{Постановка задачи и материальные параметры}

Будем считать, что волноводная структура состоит из чередующихся слоев графена и диэлектрика с толщинами $d_{g}$ и $d_{d}$. Подобная структура обладает выделенным направлением (ось $O Z$ ), что определяет одноосную анизотропию ее свойств. Толщина всей структуры равна $D=N d=N\left(d_{g}+d_{d}\right)$, где $N$ - число периодов. Тензор эффективной ДП такой среды имеет отличные от нуля компоненты $\varepsilon_{x x}=\varepsilon_{y y}=\varepsilon, \varepsilon_{z z}=\varepsilon_{0}$. ДП графена можно представить в виде $\varepsilon_{g}=i 4 \pi \sigma / \omega d_{g}$, где $\omega$ - частота, $d_{g}$ - толщина слоя, а поверхностная проводимость $\sigma=\sigma^{\prime}+i \sigma^{\prime \prime}$ является комплексной величиной. В приближении мелкослоистости указанные компоненты ДП могут быть представлены в виде

$$
\begin{gathered}
\varepsilon=\frac{\varepsilon_{d} d_{d}+\varepsilon_{g} d_{g}}{d_{g}+d_{d}}=\frac{\varepsilon_{d}+\theta \varepsilon_{g}}{\theta+1} \\
=\frac{1}{\theta+1}\left(\varepsilon_{d}-\frac{4 \pi \sigma^{\prime \prime}}{\omega d_{d}}+i \frac{4 \pi \sigma^{\prime}}{\omega d_{d}}\right), \\
\varepsilon_{0}=\frac{\varepsilon_{g} \varepsilon_{d}\left(d_{d}+d_{g}\right)}{d_{g} \varepsilon_{d}+d_{d} \varepsilon_{g}}=(1+\theta) \frac{\varepsilon_{g} \varepsilon_{d}}{\theta \varepsilon_{d}+\varepsilon_{g}},
\end{gathered}
$$

где параметр $\theta=d_{g} / d_{d}$. Если учесть, что $d_{g} \simeq 0.3 \mathrm{~nm}$ и в реальных структурах $d_{g} \ll d_{d}$, а $\theta \ll 1$, то выражения (1) фактически совпадают с выражениями, полученными в работах [15-17]:

$$
\varepsilon=\varepsilon_{d}-\frac{4 \pi \sigma^{\prime \prime}}{\omega d}+i \frac{4 \pi \sigma^{\prime}}{\omega d}, \quad \varepsilon_{z z}=\varepsilon_{d} .
$$

Слои графена обладают дисперсией, которая определяется частотной зависимостью поверхностной проводимости. Действительная и мнимая части проводимости, 


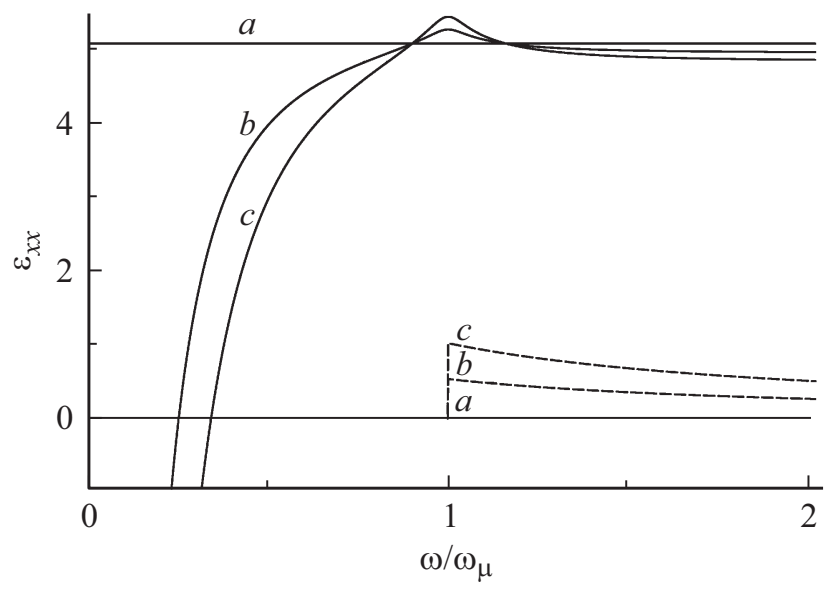

Рис. 1. Частотная зависимость действительной и мнимой частей (сплошная и штриховая линии) компоненты $\varepsilon_{x x}$ тензора эффективной ДП структуры, объемная доля графена в структуре $\theta=0,0.05,0.1$ (кривые $a, b, c)$.

согласно $[7,18,19]$, определяются выражениями

$$
\begin{gathered}
\sigma^{\prime}=\sigma_{0} \Theta(\hbar \omega-2 \mu) \\
\sigma^{\prime \prime}=\sigma_{0}\left[\frac { 1 } { 2 \pi } \left(\frac{16 k_{B} T}{\hbar \omega} \log \left(2 \operatorname{ch}\left(\frac{\mu}{2 k_{B} T}\right)\right)\right.\right. \\
\left.\left.-\log \left(\frac{(\hbar \omega+2 \mu)^{2}}{(\hbar \omega-2 \mu)^{2}+\left(2 k_{B} T\right)^{2}}\right)\right)\right]
\end{gathered}
$$

где $\sigma_{0}=e^{2} / 4 \hbar, e-$ заряд электрона, $\hbar-$ постоянная Планка, $\Theta$ - ступенчатая функция Хэвисайда [20], $k_{B}$ - постоянная Больцмана, $T-$ температура. $\mu-$ химический потенциал графена, $\mu=\hbar v_{F} \sqrt{\pi n_{0}}$, где $n_{0}$ и $v_{F}$ - концентрация носителей заряда и скорость Ферми в графене. Согласно (3), действительная часть проводимости при $\omega=\omega_{\mu}=2 \mu / \hbar$ испытывает скачок от нулевого значения до значения $\sigma_{0}$. Мнимая часть проводимости в достаточно широкой частотной области принимает отрицательные значения. Отметим возможность управления величиной химического потенциала с помощью температуры и внешнего электрического поля, что является важным моментом для практического использования рассматриваемой структуры [6]. Так, увеличение $\mu$ приводит к сдвигу скачка действительной части проводимости в область более высоких частот и понижению минимального значения мнимой части.

На рис. 1 представлена частотная зависимость действительной и мнимой частей (сплошная и штриховая линии) компоненты тензора эффективной ДП $\varepsilon$ для рассматриваемой структуры со слоями диэлектрика $\mathrm{SiO}_{2}$, для которого $\varepsilon_{d}=5.07$. В качестве расчетных параметров для графена здесь и далее используются $n_{0}=10 \mathrm{~cm}^{-2}, \mu=0.6 \mathrm{eV}, \quad v_{F}=10^{8} \mathrm{~cm} / \mathrm{s}$, $d_{g}=0.335 \mathrm{~nm}, T=300 \mathrm{~K}[6]$, частота нормирована на величину $\omega_{\mu}=1.83 \cdot 10^{15} \mathrm{~s}^{-1}$. Указанные зависимости получены для значений параметра $\theta=0.05,0.1$ (кривые $a, b, c)$. Видно, что в спектре имеется область непрозрачности структуры, где величина $\varepsilon^{\prime}$ отрицательна и объемная волна должна затухать. Однако именно в этой области в структуре возможно существование поверхностных волн. С увеличением доли графена в структуре (т.е. параметра $\theta$ ) эта область расширяется и наблюдается рост мнимой части эффективной ДП, т. е. поглощения в области $\omega>\omega_{\mu}$. В области скачка величины $\sigma^{\prime}$ (при $\omega=\omega_{\mu}$ ) действительная часть эффективной ДП достигает максимума.

Далее предполагаем, что подложка и покровная среда имеют одинаковые ДП $\varepsilon_{1}$, поэтому структура в целом является симметричной. Также считаем, что и диэлектрические среды, и графен не являются магнитоактивными, поэтому их магнитные проницаемости могут быть приняты равными $\mu_{g}=\mu_{d}=1$.

\section{Волновое поле и дисперсионное соотношение}

Пусть в структуре волна распространяется вдоль оси $O X$. При этом все компоненты волнового поля зависят от времени и продольной координаты пропорционально фактору $\exp [i(\omega t-\beta x)]$, где $\beta-$ константа распространения. В данной структуре могут распространяться волноводные моды ТЕ- и ТМ-типов. Далее мы будем рассматривать свойства только ТЕ-волн, для которых уравнения для компонент полей в структуре запишем в виде

$$
\frac{d^{2} E_{y}}{d z^{2}}+q_{j}^{2} E_{y}=0, \quad H_{z}=\frac{\beta}{k_{0}} E_{y}, \quad H_{x}=-\frac{i}{k_{0}} \frac{d E_{y}}{d z},
$$

где $j=1,2,3, q_{1,3}^{2}=-\beta^{2}+k_{0}^{2} \varepsilon_{1,3}$ и $q_{2}^{2}=\beta^{2}-k_{0}^{2} \varepsilon-$ поперечные компоненты волнового вектора вне и внутри структуры, $k_{0}=\omega / c, c-$ скорость света в вакууме. Необходимыми условиями существования волноводного режима в структуре является выполнение неравенств $\operatorname{Re}\left(q_{1,3}^{2}\right)=q_{1,3}^{\prime 2}-q_{1,3}^{\prime \prime 2}>0$, которые обеспечивают экспоненциальный спад амплитуды волнового поля при удалении от границ раздела на бесконечности. Также требуется выполнение неравенств $\beta^{\prime}>0, \beta^{\prime \prime}>0$ первое из которых указывает на положительность фазовой скорости волны в структуре, второе - на отсутствие усиления.

Решение уравнений (4) приводит к следующему распределению по координате $z$ электрического поля для симметричных $(s)$ и антисимметричных $(a)$ волноводных мод:

$$
E_{y}^{(s, a)}(z)=A_{s, a} \begin{cases} \pm G_{s, a}\left(q_{2} D / 2\right) \exp \left(q_{1} z\right), & 0<z, \\ G_{s, a}\left[q_{2}(z-D / 2)\right], & 0<z<D, \\ G_{s, a}\left(q_{2} D / 2\right) \exp \left[-q_{3}(z-d)\right], & z>D,\end{cases}
$$

где функции $G_{s}(\ldots)=\cos (\ldots), G_{a}(\ldots)=\sin (\ldots)$, а знаки \pm относятся к симметричным и антисимметричным модам соответственно; коэффициенты $A_{s, a}$ являются амплитудами поля при $z=0$, связанными с 
мощностью $P_{s, a}$, которую переносит мода на единичной ширине волновода.

Выражения для компонент магнитного поля находятся с помощью уравнений (4) при подстановке в них соотношений (5). Симметрия распределения нормальной компоненты магнитного поля $H_{z}(z)$ совпадает с симметрией поля электрического. Симметрия распределения тангенциальной компоненты $H_{x}(z)$ является противоположной симметрии электрического поля.

Для получения дисперсионного соотношения воспользуемся граничными условиями для тангенциальных компонент электрического и магнитного полей на внешних границах структуры при $z=0, D$. Так как при $z=0$ граничной поверхностью структуры является графен, обладающий высокой проводимостью, а при $z=D$ граничной поверхностью структуры является диэлектрик, то на этих границах раздела имеют место следующие граничные условия:

$$
\begin{gathered}
E_{2 y}=E_{1 y}, \quad H_{2 x}-H_{1 x}=(4 \pi \sigma / c) E_{1 y}, \\
E_{3 y}=E_{2 y}, \quad H_{3 x}=H_{2 x} .
\end{gathered}
$$

Подставляя выражения для электрического и магнитного волновых полей в граничные условия (6), приходим к следующим двум дисперсионным соотношениям для волноводных мод:

$$
\begin{gathered}
q_{2} \operatorname{tg}\left(q_{2} D / 2\right)=q_{1}+2 i \pi \sigma k_{0} / c, \\
-q_{2} \operatorname{ctg}\left(q_{2} D / 2\right)=q_{1}+2 i \pi \sigma k_{0} / c,
\end{gathered}
$$

где первое уравнение отвечает четным или симметричным по электрическому полю модам, а второе нечетным или антисимметричным.

\section{Численный анализ}

На рис. 2 представлены частотные зависимости реальной части нормированной (на величину $k_{\mu}=\omega_{\mu} / c=$ $\left.=8.67 \cdot 10^{8} \mathrm{~cm}^{-1}\right)$ константы распространения $\beta^{\prime}(\omega)$, полученные для $\mu=0.6 \mathrm{eV}, D=1 \mu \mathrm{m}$ и значений $\theta=0,0.1$. Для $\theta=0$, отвечающему случаю чисто диэлектрического заполнения, область волноводных мод ограничена двумя штриховыми линиями. Первая из этих линий $(A)$ является фотонной линией $\beta=\sqrt{\varepsilon(\omega)} \omega / c$, к которой асимптотически стремятся дисперсионные кривые с ростом частоты, вторая $(B)$ является линией отсечки (в ненормированных величинах ей отвечает зависимость $\left.\beta=\sqrt{\varepsilon_{1}} \omega / c\right)$. Для нулевой моды в этом случае отсутствует отсечка, и зависимость $\beta^{\prime}(\omega)$ практически совпадает с фотонной линией. С появлением в структуре графеновых слоев, т.е. с увеличением параметра $\theta$ для нулевой моды, появляется отсечка, а для высших мод частота отсечки смещается в область больших значений. При этом на частоте $\omega=\omega_{\mu}$ для дисперсионных кривых указанных мод возникает особенность в поведении производной $d \beta^{\prime} / d \omega$,
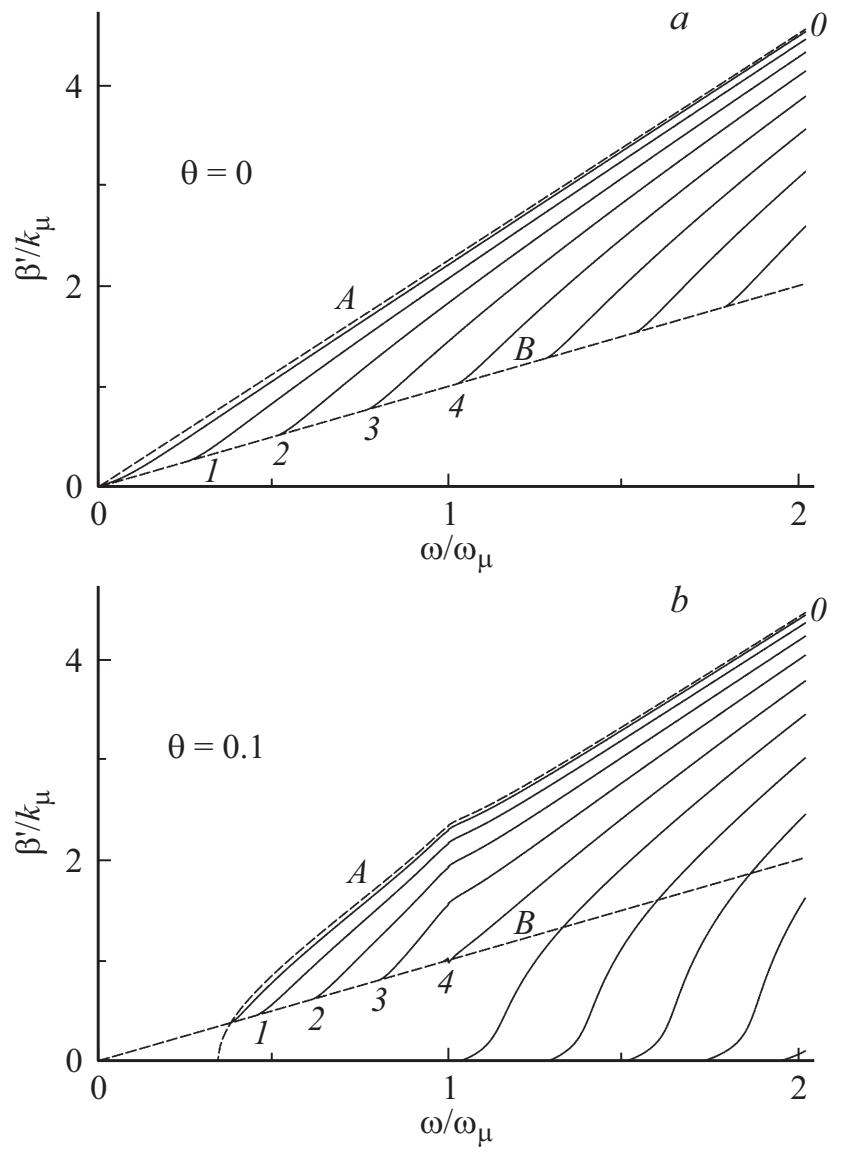

Рис. 2. Частотная зависимость действительной части константы распространения для волноводных ТЕ-мод, толщина структуры $D=1 \mu \mathrm{m}, \theta=0,0.1(a, b)$.

что отразится на групповой скорости соответствующей моды.

Число удерживаемых в структуре волноводных мод зависит как от частоты, так и от химического потенциала. Так, в области, где отсутствует поглощение (при $\left.\omega<\omega_{\mu}\right)$, при заданной толщине структуры удерживается не более четырех мод. Видно также, что при $\mu=0.6 \mathrm{eV}$ на интервале частот $\omega \leq 2 \omega_{\mu}=3.66 \cdot 10^{15} \mathrm{~s}^{-1}$ в структуре могут существовать восемь волноводных мод $(m=0,1, \ldots, 8)$, тогда как при $\mu=0.2 \mathrm{eV}$ на интервале $\omega \leq 2 \omega_{\mu}=1.22 \cdot 10^{15} \mathrm{~s}^{-1}$ в структуре реализуются только три моды $(m=0,1,2)$.

На рис. 3 приведены распределения электрического волнового поля по поперечному сечению структуры (вдоль координаты $z$ ) нулевой $\mathrm{TE}_{0}$ и первой $\mathrm{TE}_{1}$-волноводных мод при различных частотах. Видно, что распределение нулевой моды является симметричным, а первой моды - асимметричным. При этом с ростом частоты волновое поле нулевой моды все больше локализуется в центре волноводного слоя, тогда как поле первой моды локализуется вблизи границ. При удалении от границ вне слоя для обеих мод наблюдается экспоненциальный спад их амплитуд. Область 


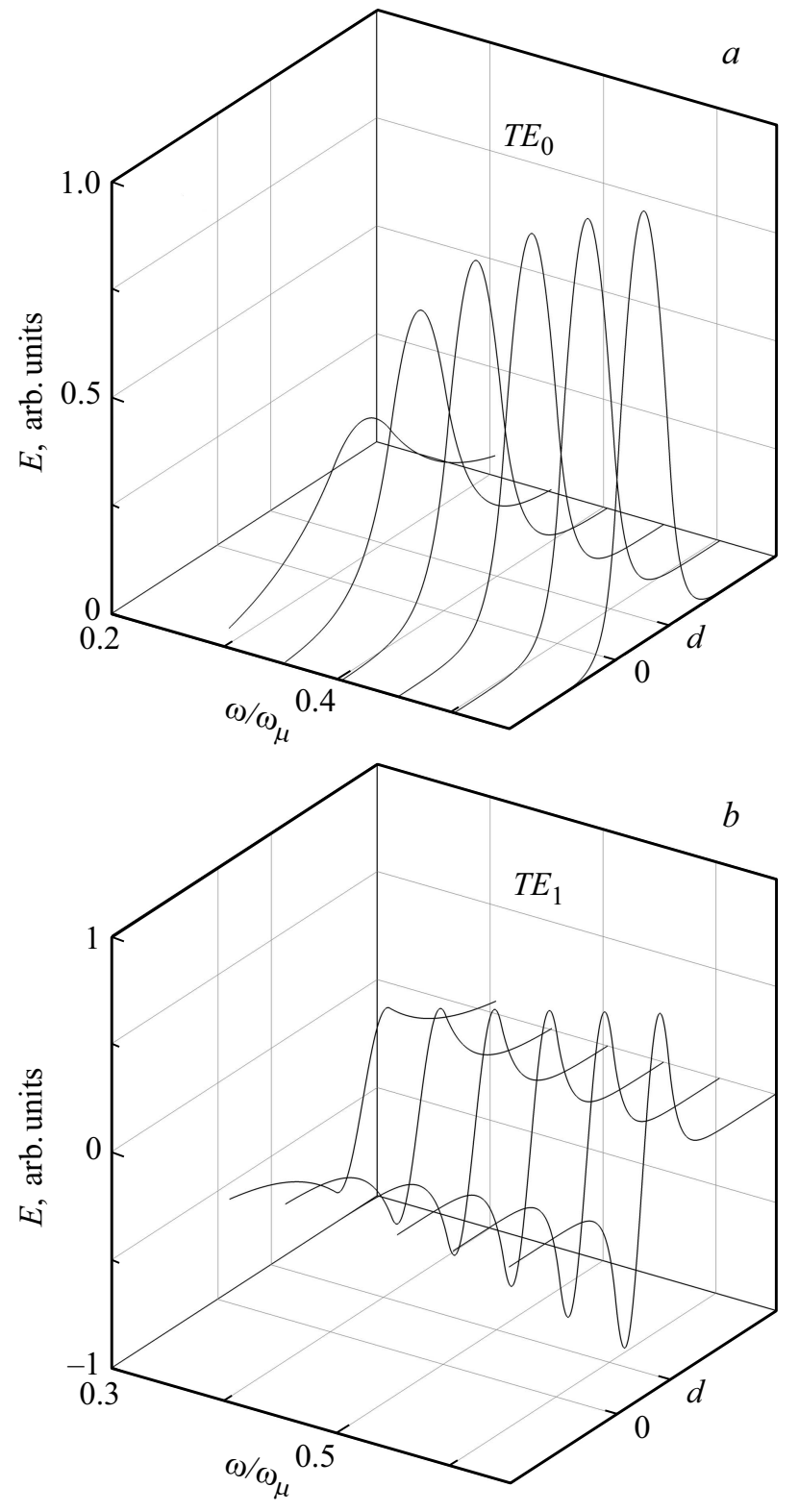

Рис. 3. Распределение электрического поля по структуре для нулевой и первой волноводных мод на разных частотах.

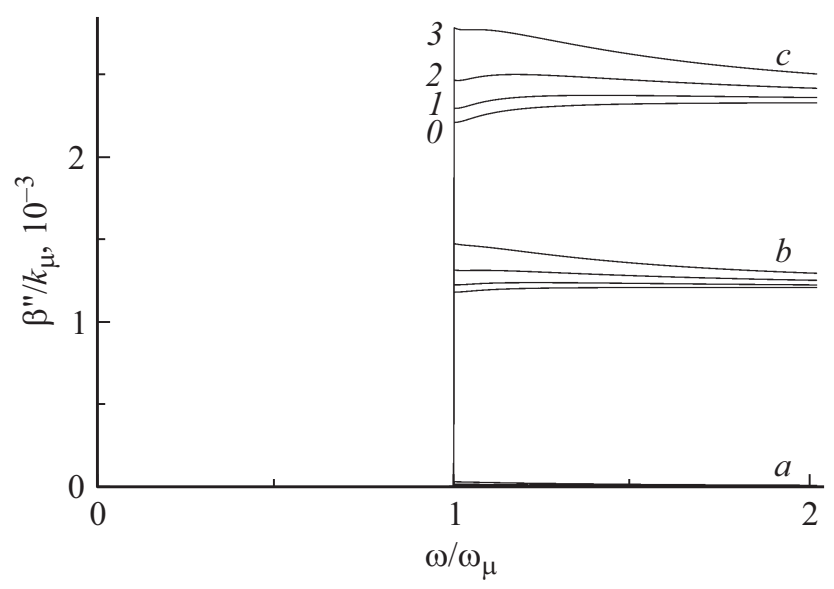

Рис. 4. Частотная зависимость мнимой части константы распространения, $\theta=0,0.05,0.1$ (кривые $a, b, c)$.
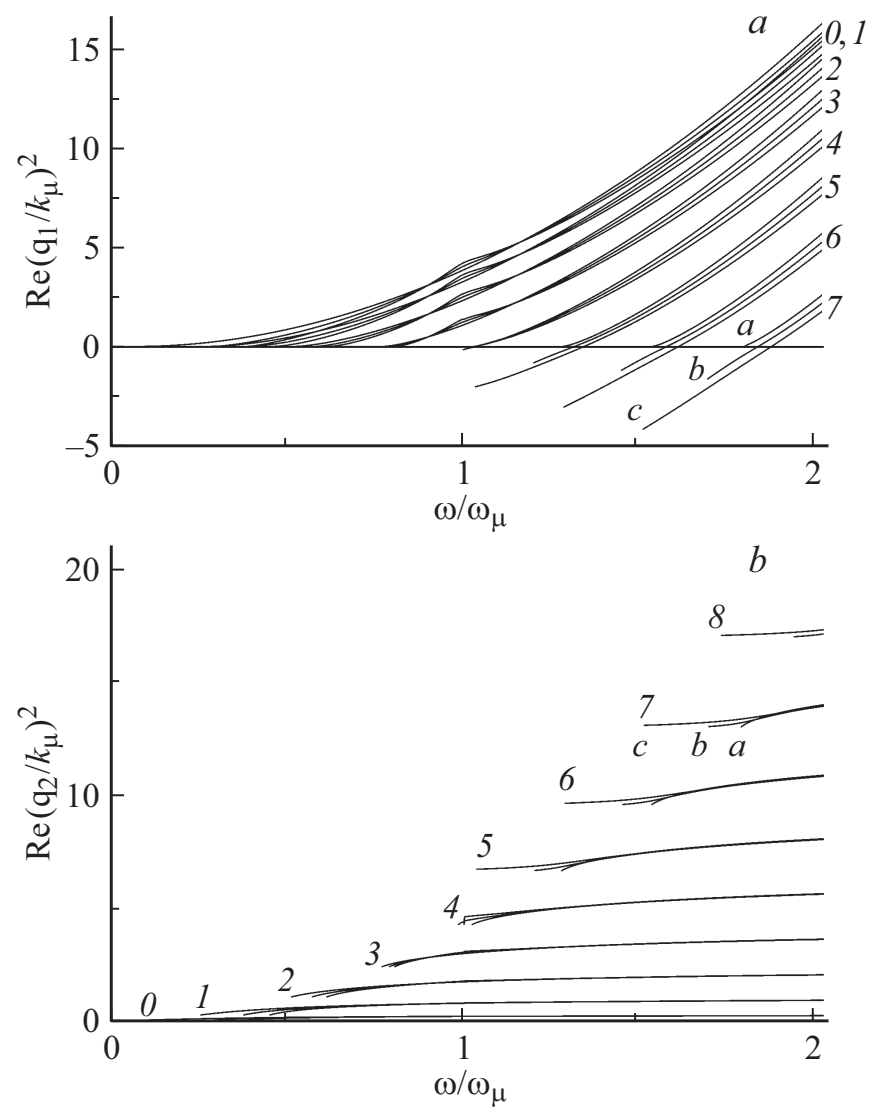

Рис. 5. Частотная зависимость действительной части величины $q^{2}$ вне и внутри структуры $(a, b), \theta=0,0.05,0.1$ (кривые $a, b, c$ ).

локализации поля моды определяется величиной, обратной действительной части поперечной компоненты волнового вектора вне структуры, т.е. $1 / q_{1,3}^{\prime}$. Отметим, что экспоненциальный спад при удалении от границ слоя наблюдается лишь для первых четырех мод (при выбранных параметрах структуры), тогда как для мод более высокого порядка распределение поля вне слоя остается периодическим, что будет показано далее.

Для мод, у которых при $\theta=0$ частота отсечки лежит выше частоты $\omega_{\mu}$, при $\theta>0$ дисперсионные кривые опускаются ниже линии отсечки $\beta^{\prime}=\sqrt{\varepsilon_{1} k_{0}}$. Для понимания такого их поведения рассмотрим частотные зависимости мнимой части константы распространения $\beta^{\prime \prime}$ волноводных мод (рис. 4), обратная величина которой определяет длину пробега моды, $l \approx 1 / \beta^{\prime \prime}$. Видно, что затухание на частотах $\omega<\omega_{\mu}$ для всех волноводных мод отсутствует, тогда как при $\omega>\omega_{\mu}$ затухание появляется и его уровень существенно зависит от параметра $\theta$, т.е. числа слоев графена при данной толщине структуры. С ростом $\theta$ величина $\beta^{\prime \prime}$ для каждой последующей моды постепенно растет, достигая максимума, но с дальнейшим ростом частоты затухание уменьшается, приближаясь к значению, отвечающему уровню затухания нулевой моды. 
Рассмотрим теперь частотную зависимость действительной части квадрата поперечной компоненты волнового вектора вне и внутри структуры, т.е. $\operatorname{Re} q_{1,2}^{2}(\omega)$, которая приведена на рис. 5. Видно, что для дисперсионных кривых, лежащих ниже линии отсечки при $\theta>0$ и $\omega>\omega_{\mu}$, вне волновода выполняется неравенство $\operatorname{Re} q_{1}^{2}<0$, и, следовательно, соответствующие моды уже не являются локализованными. Однако поперечное распределение волнового поля внутри волновода для этих мод является периодическим, так как выполняется неравенство $\operatorname{Re} q_{2}^{2}>0$. Поэтому указанные моды в целом являются излучательными, хотя и имеют дискретный спектр.

На рис. 6 представлены дисперсионные зависимости $\beta^{\prime}(\omega)$, полученные при $\theta=0.1$ для структур разной толщины $(a)$, а также при $D=1 \mu \mathrm{m}$ и разных значениях параметра $\theta(b)$. На рисунке $a$ кривые $1-4$ отвечают нулевой моде при $D=25,50,100,150 \mathrm{~nm}$. Видно, что для первых трех толщин структуры в рассматриваемом интервале частот возможно существование только нулевой моды. Лишь для структуры с $D=150 \mathrm{~nm}$ в области частот $\omega>1.75 \omega_{\mu}$ наряду с нулевой модой появляется и первая мода (кривая 5). На рисунке $b$ кривые $a, b, c, d, e, f$ отвечают значениям параметра $\theta=0,0.05,0.06,0.07$,
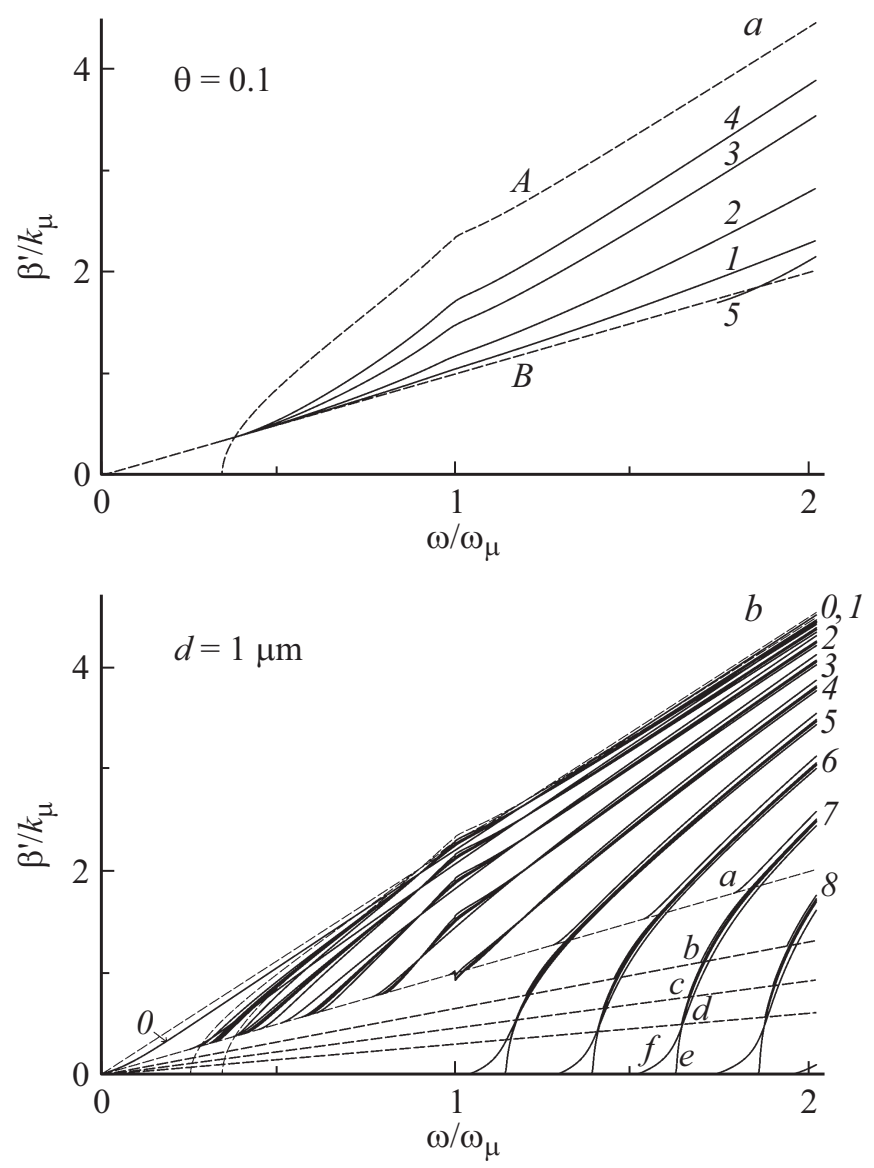

Рис. 6. Дисперсионные зависимости $\beta^{\prime}(\omega)$ для структур с параметрами $\theta=0.1$ и $D=25,50,100,150 \mathrm{~nm}(a) ; D=1 \mu \mathrm{m}$ и $\theta=0,0.05,0.06,0.065,0.07,0.1$ ( $b$, кривые $a, b, c, d, e, f)$.
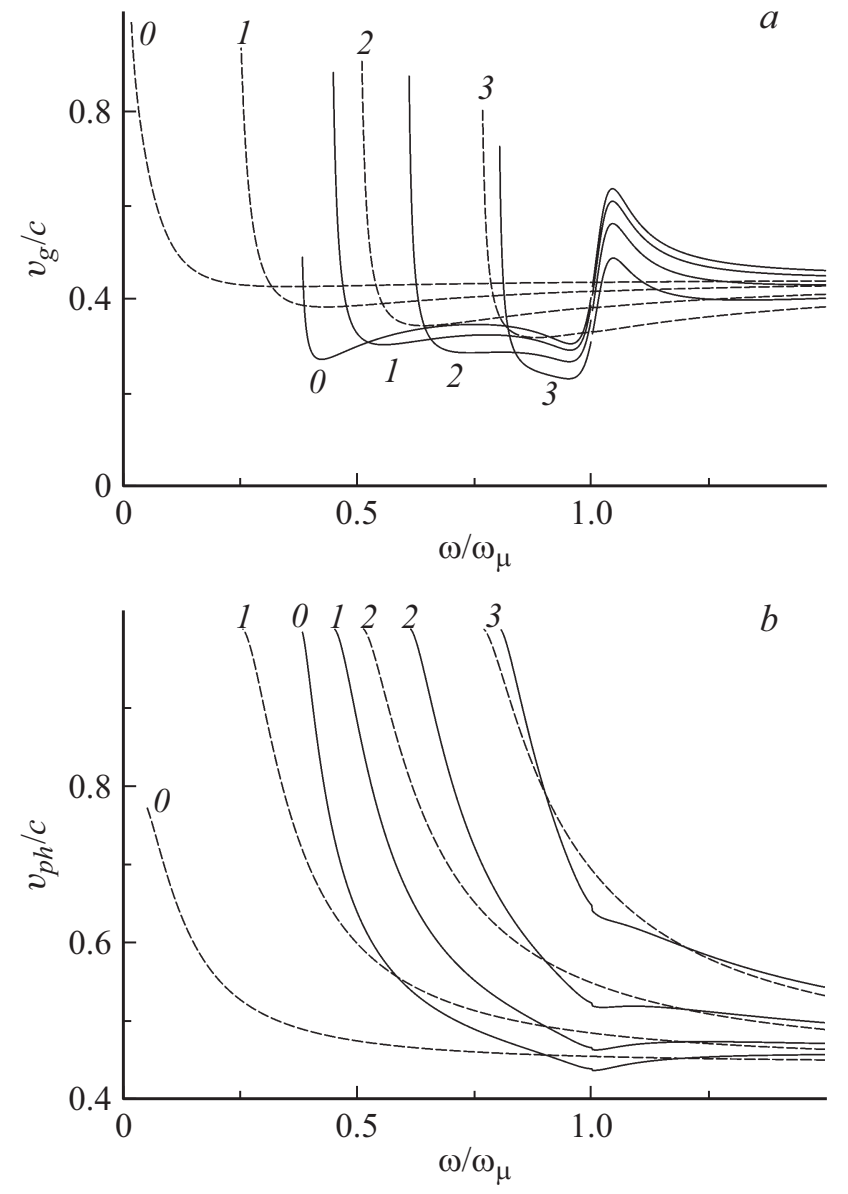

Рис. 7. Групповая и фазовая скорости волноводных мод в структуре с $D=1 \mu \mathrm{m}$ и $\theta=0,0.1$ (штриховые и сплошные кривые).

0.1, а числа справа обозначают номера мод. Видно, что с увеличением доли графена в волноводной структуре наблюдается увеличение области значений константы распространения, в которой мода при $\omega>\omega_{\mu}$ является излучательной.

На рис. 7 приведены частотные зависимости групповой и фазовой скоростей первых четырех волноводных мод для структуры с $\theta=0$ и $\theta=0.1$ (штриховая и сплошные кривые). При $\theta=0$ указанные зависимости практически не отличаются от аналогичных зависимостей для планарного диэлектрического волновода, что говорит о слабом влиянии двух графеновых обкладок на волноводные свойства структуры, толщина которой намного больше толщины графенового слоя. Как уже отмечалось, для структуры с $\theta \neq 0$ частота отсечки всех мод увеличивается по сравнению со случаем $\theta=0$. При $\omega=\omega_{\mu}$ имеют место скачок поглощения, а также особенность в поведении производной $d \beta^{\prime} / d \omega$, что отражается на поведении групповой скорости соответствующих мод. Так, для представленных мод имеются достаточно глубокие минимумы групповой скорости с $v_{g} \simeq(0.23-0.27) c$. В диапазоне $\omega=(0.9-1.1) \omega_{\mu}$ ха- 
рактер изменения групповых скоростей мод близок к резонансному, что связано с появлением у графена проводимости и наличием максимума диэлектрической проницаемости в этой области. При дальнейшем росте частоты скорости всех мод приближаются к скорости $v_{g} \simeq 0.45 c$.

Кривые фазовых скоростей также испытывают смещение вправо и скачок на частоте $\omega_{\mu}$. В целом при прочих равных условиях волноводные моды в эффективной среде медленнее, чем в чистом диэлектрике. В обоих случаях с ростом номера моды замедление проявляется сильнее, но при дальнейшем росте частоты выходят на один и тот же уровень. Для каждой моды фазовая скорость не обладает особенностями и плавно уменьшается от $c$ до $0.44 c$.

\section{Заключение}

В работе исследованы особенности распространения волноводных мод ТЕ-поляризации в плоско-слоистой структуре конечной толщины, состоящей из чередующихся слоев диэлектрика и графена, обладающего высокой плоскостной проводимостью. Граничными поверхностями структуры являются два слоя графена. В приближении эффективной среды для различных значений отношения толщин соседних слоев в структуре получены частотные зависимости компонент тензора эффективной ДП структуры, распределения волнового поля по сечению волновода, групповой и фазовой скоростей. Численный анализ дисперсионного соотношения показал, что на частотах $\omega<\omega_{\mu}$ (характерная частота $\omega_{\mu}=2 \mu / \hbar$ определяется химическим потенциалом графена) в структуре отсутствует поглощение даже при комнатной температуре. При $\omega=\omega_{\mu}$ имеют место скачок поглощения, а также особенность в поведении производной $d \beta^{\prime} / d \omega$ на дисперсионных кривых волноводных мод, что отражается на поведении групповой скорости соответствующих мод. С ростом доли графена в структуре появляется отсечка нулевой моды и сдвиг частоты отсечки высших мод в высокочастотную область, увеличивается поглощение в структуре и появление дискретных излучательных мод. Групповая скорость мод в исследуемом частотном интервале положительна, при максимально возможном замедлении скорость мод составляет $v_{g} \simeq 0.23 c$.

Работа выполнена при поддержке Министерства образования и науки РФ в рамках проектов № 3.6825.2017/БЧ и 14.Z50.31.0015.

\section{Список литературы}

[1] Морозов С.В., Новоселов К.С., Гейм А.К. // УФН. 2008. T. 178 . C. 776.

[2] Kechedzhi K., Kashuba O., Fal'ko V.I. // Phys. Rev. B. 2008. V. 77. P. 193403.
[3] Castro Neto A.H., Guinea F., Peres N.M.R., Novoselov K.S., Geim A.K. // Rev. Mod. Phys. 2009. V. 81. P. 109.

[4] Shemella P., Nayak S.K. // Appl. Phys. Lett. 2009. V. 94. P. 032101.

[5] Falkovsky L.A., Pershoguba S.S. // Phys. Rev. B. 2007. V. 76. P. 153410.

[6] Falkovsky L.A. // J. Phys.: Conf. Ser. 2008. V. 129. P. 012004.

[7] Katsnelson M.I. // Europhys. Lett. 2008. V. 84. P. 37001.

[8] Berman O.L., Kezerashvili R.Ya. // J. Phys.: Cond. Mat. 2012. V. 24. P. 015305.

[9] Madani A., Entezar S.R. // Phys. B. 2013. V. 431. P. 1.

[10] Колесников А.А., Лозовик Ю.Е. // Труды МФТИ. 2013. T. 5(1). C. 53.

[11] El-Naggar A.S. Tunable Terahertz Omnidirectional Photonic Gap in One Dimentional Grapheme-based Photonic Crystals. Springer Science, 2014.

[12] Liang H., Ruan S., Zhang M., Su H., Ling Li I. // Appl. Phys. Lett. 2015. V. 107. P. 091602.

[13] Gan C.H., Chu H.S., Li E.P. // Phys. Rev. B. 2012. V. 85. P. 125431.

[14] Zhu B., Ren G., Zheng S., Lin Z., Jian S. // Opt. Express. 2013. V. 21. P. 17089.

[15] Svintsov D., Vyurkov V., Ryzhii V., Otsuji T. // arXiv: 2012. 1211. 3629.

[16] Белоненко М.Б., Лебедев Н.Г., Янюшкина Н.Н. // ФТТ. 2012. T. 54(1). C. 162.

[17] Буслаев П.И., Иорш И.В., Шадривов И.В., Белов П.А., Кившарь Ю.С. // Письма в ЖЭТФ. 2013. Т. 97. С. 619.

[18] Falkovsky L.A., Varlamov A.A. // Eur. Phys. J. B. 2007. V. 56. P. 281.

[19] Vasko F.T., Ryzhii V. // Phys. Rev. B. 2007. V. 76. P. 233404.

[20] Справочник по специальным функциям. / Под ред. Абрамовица М., Стиган И. М.: Наука, 1979. 